\title{
XXVII.
}

\section{Bemerkung zu dem Aufsatz über die elektrische Entartnngsreaction.}

\author{
Von \\ Dr. Frusbery.
}

Die in dem Aufsatze S. 244 ausgeführte Hypothese über den Molekularvorgang bei der Contraction des Muskels, insbesondere die Heranziehung einer peripolar elektrischen Beschaffenhait der Muskelmolekeln zur Deutung gewisser Theilerscheinungen bei der galvanischen Reizung des Muskels konnte im besten Fall nur ein Bild und eine Formel zu sein beansprachen, deren $Z$ weck und Werth darauf hinauslief, einen gemeinsamen Gesichtspunkt aufzustellen, von welchem sich die uns ihrem Nebeneinander nach bekannten Thatsachen ableiten und in ihren gleichmăssigen Bedingungen erkennen lassen sollten.

Als ich neuerdings, zum $Z$ weck eines Vortrages in der Versammlang des psychiatrischen Vereins der Rheinprovinz am 9. November d. J. diese theoretischen Dinge wieder aufnahm, glaube ich glücklicher gewesen und zu Anschauungen gekommen zu sein, die nicht ein blosses Bild, sondern ein Stūck des wirklichen innern Geschehens bei der Muskelcontraction bieten. Leider kann aus äusseren Gründen die Darlegung dieser modificirten Anschauungen erst im nächsten Hefte dieses Archivs zum Abdruck kommen. Ich muss mich daher auf die vorläufige Mittheilung beschrănken, dass es zur Erklärung der sămmtlichen in Frage kommenden Erscheinungen der Annahme einer peripolaren Beschaffenheit der Molekeln nicht bedarf, und dass das mit den Vitalitäts- resp. Activitătszuständen der Molekel verschiedene elektrische Verhalten nicht von Bedeutung ist für den Molekularmechanismus des Contractionsvorganges. Die Ausführungen der obigen Arbeit, die auf der peripolaren Beschaffenheit der Molekeln basiren, kann ich also nicht mehr als zutreffende bezeichnen. Andernach, den 6. December 1878. 